\title{
Radiofrequency ablation
}

Leonie Scholtz MB ChB, MMedRad (D)

Department of Radiology University of Pretoria
The exciting developments in magnetic resonance imaging are leading us 1 towards the fetus, the female breast and the prostate gland, resulting in a smiling contented look on the face of radiology, not to mention the prospect of regaining imaging of the coronary arteries (that has been the sole and dearly cherished possession of the cardiologists for decades), with multi-slice computed tomography approaching resolution comparable to digital angiography. Despite all the commotion surrounding the MR and CT consoles we can always rely on the interventional magicians to invent new fascinating, sometimes frightening and occasionally totally impractical procedures to stimulate the everlasting repressed surgical pulses in our blood. We have dilated, embolised, occluded, excluded, infiltrated and biopsied almost everything. With radiofrequency ablation (RFA), 'cooking' is definitely the new kid on the block. RFA has been employed for some time for the treatment of primary and metastatic liver tumours. Pancreatic tumours, bone metastases and intra-pulmonary tumours are being treated similarly and RFA has recently entered phase 2 clinical trials for the treatment of small renal tumours.

RFA involves the use of high-frequency electrical current to cause heat-based tissue destruction through needle electrodes of varying diameters depending on the size of the tumour treated. Limitations include tumours larger than $5 \mathrm{~cm}$ in diameter as well as proximity to vascular structures and other visceral organs. Treatment of liver tumours can be done percutaneously or as an open procedure during which the tumour can be visualised with an ultrasound probe placed directly on the surface of the liver.

RFA is still in its infancy. The initial results are promising and have certainly caught the imagination of interventional radiologists, surgeons and oncologists. The length of treatment varies considerably, depending on the size and number of lesions treated. It would seem that we are facing long sessions of coagulating various lesions, hopefully with excellent results, whilst in the process broadening our musical repertoire, listening to Bach, the Beatles or Britney, depending on the age of the interventional radiologist hanging on to the end of the electrode.

\section{Leonie Scholtz}

Editor 